\title{
Fractionalized Fermi liquids
}

\author{
T. Senthil ${ }^{(1)}$, Subir Sachdev ${ }^{(2)}$, and Matthias $\operatorname{Vojta}^{(3)}$ \\ (1) Department of Physics, Massachusetts Institute of Technology, Cambridge MA 02139 \\ (2) Department of Physics, Yale University, P.O. Box 208120, New Haven CT 06520-8120 \\ (3) Institut für Theorie der Kondensierten Materie, Universität Karlsruhe, Postfach 6980, D-76128 Karlsruhe, Germany
}

(October 30, 2018)

\begin{abstract}
In spatial dimensions $d \geq 2$, Kondo lattice models of conduction and local moment electrons can exhibit a fractionalized, non-magnetic state (FL*) with a Fermi surface of sharp electron-like quasiparticles, enclosing a volume quantized by $\left(\rho_{a}-1\right)(\bmod 2)$, with $\rho_{a}$ the mean number of all electrons per unit cell of the ground state. Such states have fractionalized excitations linked to the deconfined phase of a gauge theory. Confinement leads to a conventional Fermi liquid state, with a Fermi volume quantized by $\rho_{a}(\bmod 2)$, and an intermediate superconducting state for the $Z_{2}$ gauge case. The $\mathrm{FL}^{*}$ state permits a second order metamagnetic transition in an applied magnetic field.
\end{abstract}

The physics of the heavy fermion metals, intermetallic compounds containing localized spin moments on $d$ or $f$ orbitals and additional bands of conduction electrons, has been of central interest in the theory of correlated electron systems for several decades [1, 2, 3, 3]. These systems are conveniently modelled by the much studied Kondo lattice Hamiltonian, in which there are exchange interactions between the local moments and the conduction electrons, and possibly additional exchange couplings between the local moments themselves. To be specific, one popular Hamiltonian to which our results apply is:

$$
\begin{aligned}
H= & -\sum_{j, j^{\prime}} t\left(j, j^{\prime}\right) c_{j \sigma}^{\dagger} c_{j^{\prime} \sigma}+\frac{1}{2} \sum_{j} J_{K}(j) \vec{S}_{j} \cdot c_{j \sigma} \vec{\tau}_{\sigma \sigma^{\prime}} c_{j \sigma^{\prime}} \\
& +\sum_{j<j^{\prime}} J_{H}\left(j, j^{\prime}\right) \vec{S}_{j} \cdot \vec{S}_{j^{\prime}} .
\end{aligned}
$$

Here the local moments are $S=1 / 2$ spin $\vec{S}_{j}$, and the conduction electrons $c_{j \sigma}(\sigma=\uparrow \downarrow)$ hop on the sites $j, j^{\prime}$ of some regular lattice in $d$ spatial dimensions with amplitude $t\left(j, j^{\prime}\right), J_{K}>0$ are the Kondo exchanges $(\vec{\tau}$ are the Pauli matrices), and explicit short-range Heisenberg exchanges, $J_{H}$, between the local moments have been introduced for theoretical convenience. A chemical potential for the $c_{\sigma}$ fermions which fixes their mean number at $\rho_{c}$ per unit cell of the ground state is implied. We have not included any direct couplings between the conduction electrons as these are assumed to be well accounted by innocuous Fermi liquid renormalizations.

For simplicity, we restrict our attention here to nonmagnetic states, in which there is no average static moment on any site $\left(\left\langle\vec{S}_{j}\right\rangle=0\right)$, and the spin rotation invariance of the Hamiltonian is preserved: the $\vec{S}_{j}$ moments have been 'screened', either by the $c_{\sigma}$ conduction electrons, or by their mutual interactions (there is a natural extension of our results to magnetic states). It is widely accepted [1, 3, 4, 5, 6, 6, 7] that such a ground state of $H$ is a conventional Fermi liquid (FL) with a Fermi surface of 'heavy' quasiparticles, enclosing a volume, $\mathcal{V}_{F L}$ determined by the Luttinger theorem:

$$
\mathcal{V}_{F L}=\mathcal{K}_{d}\left[\rho_{a}(\bmod 2)\right] .
$$

Here $\mathcal{K}_{d}=(2 \pi)^{d} /\left(2 v_{0}\right)$ is a phase space factor, $v_{0}$ is the volume of the unit cell of the ground state, $\rho_{a}=n_{\ell}+\rho_{c}$ is the mean number of all electrons per volume $v_{0}$, and $n_{\ell}$ (an integer) is the number of local moments per volume $v_{0}$. Note that $\rho_{c, a}$ need not be integers, and the $(\bmod 2)$ in (2) allows neglect of fully filled bands. In $d=1$, (2) has been established rigorously by Yamanaka et al. [5]. In general $d$, a non-perturbative argument for (2) , assuming that the ground state is a Fermi liquid, has been provided by Oshikawa [6], who also emphasized that that the Luttinger theorem can be regarded as a "quantization" of $\mathcal{V}_{F L}$.

The primary purpose of this paper is to show that there exist non-magnetic, metallic states $\left(\mathrm{FL}^{*}\right)$ in dimensions $d \geq 2$ with a Fermi surface of ordinary $S=1 / 2$, charge $-e$, sharp quasiparticles, enclosing a volume

$$
\mathcal{V}_{F L^{*}}=\mathcal{K}_{d}\left[\left(\rho_{a}-1\right)(\bmod 2)\right]
$$

over a finite range of parameters. For $n_{\ell}=1 \mathcal{V}_{F L^{*}}$ is determined by the density of conduction electrons alone. A number of earlier works [8,95:10] have considered a Fermi surface of conduction electrons alone, decoupled in meanfield from the local moments. Here we establish the conditions under which (3) characterizes a stable phase of matter for generic couplings, beyond simple decoupled models. One of our findings is that any $\mathrm{FL}^{*}$ state must be fractionalized [11] i.e. it possesses $S=1 / 2$ neutral spinon excitations (which are entirely distinct from the Fermi surface quasiparticles) which carry a charge under a gauge group which characterises the topological order in the $\mathrm{FL}^{*}$ state. We will consider here only the simplest case of a $Z_{2}$ gauge group [11,12], in which case the $Z_{2} \mathrm{FL}^{*}$ state possesses a gap to topologically non-trivial 'vison' states 11, 13, 14, which carry $Z_{2}$ flux. The connection with a $Z_{2}$ (or other) gauge theory explains why the $\mathrm{FL}^{*}$ is not possible in $d=1$ : a translationally invariant deconfined phase of the gauge theory is only present for $d \geq 2$. We will also discuss the quantum transition between the $Z_{2} \mathrm{FL}^{*}$ state and the conventional FL state 
as the exchange couplings are varied: this transition is preempted by a superconducting state.

We note that the $\mathrm{FL}^{*}$ state does not contradict the non-perturbative computation by Oshikawa [6] of $\mathcal{V}_{F L}$; on the contrary, this argument helps establish the intimate connection between (3) and topological order. Oshikawa placed the system on a torus, and considered the adiabatic evolution of the ground state upon threading a magnetic flux of $h c / e$ felt by the electrons with spin up (in some basis) through one of the holes of the torus. For insulating antiferromagnets with a fractionalized spin liquid ground state (a resonating valence bond (RVB) state), this procedure connects two of the topologically distinct states which become degenerate in the thermodynamic limit in a toroidal geometry [14, 15, 16] i.e. it connects states with and without a vison threading the hole of the torus. The $\mathrm{FL}^{*}$ state of the Kondo lattice models we are discussing here has a similar topological order, and the toroidal system has global vison excitations which are degenerate with the ground state in the thermodynamic limit. Oshikawa did not consider such excitations, and only included the electron-like Fermi surface quasiparticles. Consequently, his argument does not directly apply to the $\mathrm{FL}^{*}$ state, and a modification accounting for vison excitations shows that the volume $\mathcal{V}_{F L^{*}}$ is allowed. In other words, the Fermi volume is still quantized, but differently from that in a Fermi liquid.

The volume $\mathcal{V}_{F L}$ is observed in many compounds, and in particular in those with weak direct exchange $J_{H}$ between different local moments. Doniach [17 pointed out that increasing $J_{H}$ would lead to magnetically ordered states. However, the effective exchange interactions between the local moments are strongly frustrated in many common lattices, so that the magnetic order may be very fragile or entirely absent: it is these frustrated systems which are favorable candidates for displaying a non-magnetic $\mathrm{FL}^{*}$ state. The generic appearance of superconductivity in the crossover between the $Z_{2} \mathrm{FL}^{*}$ and FL states is experimentally significant: this may be regarded as a proposed 'mechanism' for superconductivity in heavy fermion systems, which bears some similarity to the RVB theory [18]. The critical temperature $(T)$ for the onset of superconductivity, $T_{c}$, can be small.

The $T>0$ behavior of the $Z_{2} \mathrm{FL}^{*}$ state depends on $d$, as discussed for other fractionalized states in Ref. [11]. In $d=3$ there is a finite temperature phase transition associated with the onset of topological order. This is absent in $d=2$ where the topological order is present only at $T=0$. In layered quasi-two dimensional materials, both types of behavior (corresponding to two distinct $T=0$ phases) are possible.

To understand the origin of our results in the context of (1), consider first the limiting case $J_{K}=0$, when the $c_{\sigma}$ fermions and the $\vec{S}_{j}$ spins are decoupled. While the $c_{\sigma}$ fermions will occupy states inside a Fermi surface enclos- ing volume $\mathcal{K}_{d}\left[\rho_{c}(\bmod 2)\right]$, there are two distinct classes of possibilities for a non-magnetic ground state for the $\vec{S}_{j}$ spins interacting via $J_{H}$.

The first is a ground state with confinement of spinons and a unit cell with $n_{\ell}$ even; this may require breaking of translational symmetry by the appearance of bond order 19. In this case $\rho_{a}=\rho_{c}(\bmod 2)$, and turning on a finite $J_{K}$ leads to a FL state, possibly with co-existing bond order, with the Fermi volume $\mathcal{V}_{F L}$ equal to that at $J_{K}=0$.

The second possibility, of central interest in this paper, is that $\vec{S}_{j}$ moments form a fractionalized spin liquid ground state with $n_{\ell}$ odd [11, 12,20]: this happens on frustrated lattices, as has been supported by studies [21] on the triangular lattice. A fundamental property of such a state is its topological stability [11], and the associated gap towards creation of vison excitations which carry unit flux of a $Z_{2}$ gauge field. The $S=1 / 2$ spinon excitations above this state carry a unit $Z_{2}$ gauge charge. Now turn on a small $J_{K} \neq 0$. The key argument of this paper is that the resulting ground state is smoothly connected to the $J_{K}=0$ limit: the quantum numbers of the latter state and its excitations are topologically protected, the vison gap will survive for a finite range of $J_{K}$ values, and perturbation theory in powers of $J_{K}$ is nonsingular. So we obtain our advertised $\mathrm{FL}^{*}$ state, with a Fermi surface of spin-1/2, charge $-e$, quasiparticles enclosing the volume $\mathcal{V}_{F L^{*}}$ equal to that at $J_{K}=0$, along with a separate set of spin- $1 / 2$ neutral spinon excitations 222. Physically, each local moment has formed a singlet with another local moment in an RVB spin liquid state - the Kondo coupling with the conduction electrons is ineffective in breaking these singlets. The Fermi surface quasiparticles have a weak residual interaction, arising from exchanges of pairs of spinons, which could lead to their pairing in a high angular momentum channel at some very low $T$ : this produces an exotic superconductor which co-exists with a fractionalized spin liquid 23] which we will not discuss further - the superconductivity discussed elsewhere in this paper is more robust and a qualitatively different state.

As we continue to increase $J_{K}$, the physics of the Kondo effect will eventually be manifest: it will become favorable for a local moment to form a Kondo singlet with the conduction electrons rather than with other local moments. This may be formalized as follows, for the case in which the spinons are fermions: Representing the $\vec{S}_{j}$ moments by $S=1 / 2$ fermions $f_{j \sigma}\left(\vec{S}_{j}=f_{j \sigma}^{\dagger} \vec{\tau}_{\sigma \sigma^{\prime}} f_{j \sigma^{\prime}} / 2\right.$ with the single-occupancy constraint $f_{j \sigma}^{\dagger} f_{j \sigma}=1$ ), the formation of Kondo singlets is signaled by a non-zero hybridization between the $f_{\sigma}$ and the $c_{\sigma}$ fermions. This can be expressed more precisely in terms of the composite boson fields $B_{1}=f_{\sigma}^{\dagger} c_{\sigma}$ and $B_{2}=\varepsilon^{\sigma \sigma^{\prime}} f_{\sigma} c_{\sigma^{\prime}}$, where $\varepsilon$ is the antisymmetry tensor with $\varepsilon^{\uparrow \downarrow}=1$. Both of these fields have a unit $Z_{2}$ gauge charge, an electromagnetic 
charge $e$ and are spin singlet: Condensation of these bosons implies a non-zero amplitude that a local moment has formed a Kondo singlet with the conduction electrons. This condensation indicates that the $Z_{2}$ gauge theory enters a Higgs phase which can also be identified with a phase in which $Z_{2}$ charges are confined [24. Moreover, as the spinon pairing amplitude $\left\langle\varepsilon^{\sigma \sigma^{\prime}} f_{\sigma} f_{\sigma^{\prime}}\right\rangle$ is generically non-zero in the small $J_{K}$ fractionalized phase [11], the condensation of $B_{1}$ implies condensation of $B_{2}$ (and vice-versa), and there is only a single $Z_{2}$ confinement transition. More importantly, the pairing of the spinons and the condensation of $B_{1,2}$ implies that the resulting phase also has pairing of the conduction electrons, and is a superconductor at $T=0$.

Consider now the behavior when $J_{K}, t \gg J_{H}$. In the limit $J_{H}=0$, the usual FL state is expected (at least at generic incommensurate conduction electron density). Turning on a weak non-zero $J_{H}$ potentially introduces a weak instability toward superconductivity, as will be the case in our mean-field theory below. However, the FL state may still be stabilized by a weak nearest neighbor repulsive interaction between the conduction electrons.

The general considerations above can be illustrated by a simple mean-field computation of the phase diagram of $H$. We applied the large $N$ method associated with a generalization of $H$ to $\operatorname{Sp}(N)$ symmetry [25] on the triangular lattice. It is important to note that both the symmetry group and the lattice have been carefully chosen to allow for a mean field state with $Z_{2}$ topological order, stable under gauge fluctuations [12]; in particular, there are topologically distinct mean-field ground states in a toroidal geometry, differing in the $Z_{2}$ flux through the holes of the torus. Other choices [\$] for the lattice or the symmetry group lead to mean-field solutions which are generically disrupted by $\mathrm{U}(1)$ or $\mathrm{SU}(2)$ gauge fluctuations in $d=2$. We used self-conjugate, fully antisymmetric (fermionic) representations for the spin states, and the computations were then similar to earlier work on the $t$ - $J$ model 25]. For $J_{K}=0$ and nearest neighbor $J_{H}$, these representations yields globally stable solutions in which the $\vec{S}_{j}$ spins are paired in fully dimerized states which break lattice symmetries. As we are not interested in such states here, we restricted our analysis to saddle points which preserve all lattice symmetries. Such RVB saddle points can be stabilized by additional couplings between the local moments; they are also stable for nearest-neighbor $J_{H}$ for bosonic spin representions [26,25, but these, unfortunately, do not allow a simple description of the FL state at large $J_{K}$. It is possible that the spinons undergo a change from bosonic to fermionic statistics with increasing $J_{K}$ within the $\mathrm{FL}^{*}$ state, but this will not be captured by our present mean field theory which has only fermionic spinons.

The phase diagram is shown in Fig 1 as a function of $J_{K}$ and $T$ for fixed $J_{H}, t$, and $\rho_{c}$.

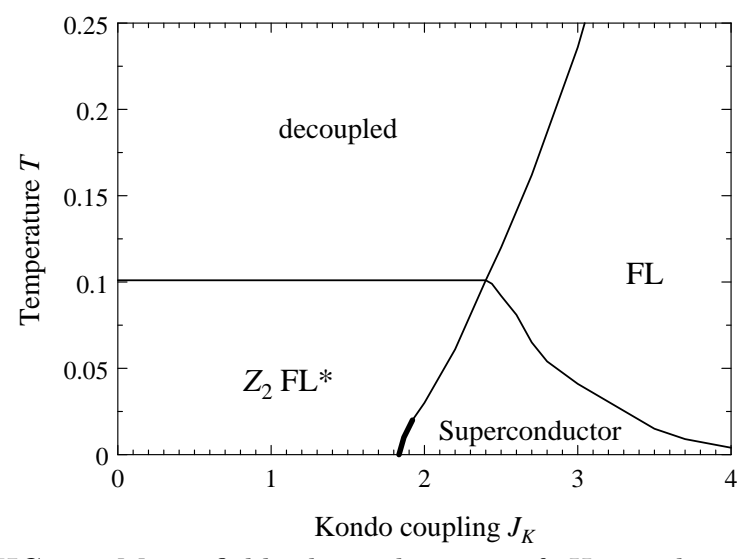

FIG. 1. Mean field phase diagram of $H$ on the triangular lattice. We used fermionic representations of $\operatorname{Sp}(N)$ for the spins, and restricted attention, by hand, to saddle points which preserve all lattice symmetries. We had nearest-neighbor $t=1, J_{H}=0.4$, and $\rho_{c}=0.7$. The superconducting $T_{c}$ is exponentially small, but finite, for large $J_{K}$, while it is strictly zero for small $J_{K}$. Thin (thick) lines are second (first) order transitions. The transitions surrounding the superconductor will survive beyond mean field theory, while the others become crossovers.

In addition to the $Z_{2} \mathrm{FL}^{*}$ and $\mathrm{FL}$ states, and an intermediate superconducting state, whose character we have already discussed, there is also a high temperature "decoupled" state. Here, in the mean field saddle point, the spins are mutually decoupled from each other, and from the conduction electrons. This decoupling is, of course, an artifact of the saddle point, and it points to a regime where all excitations are incoherent but strongly interacting with each other. For the case where the superconducting phase is present only at very low temperatures (as may well be the case beyond mean field theory), this incoherent regime represents the quantum-critical region of the $Z_{2} \mathrm{FL}^{*}$-FL transition. A separate description of this incoherent quantum critical dynamics was provided by the large-dimensional saddle point studied by Burdin et al. [10], where it was related to the gapless spin liquid state of Ref. 27].

An interesting $T=0$ quantum phase transition appearing in Fig 1 is that between the $\mathrm{FL}^{*}$ and superconducting states. As we discussed earlier, this transition is associated with the condensation of the charge $e$ bosons $B_{1,2}$. A critical theory of the transition can be written down in terms of $B_{1,2}$ and the conduction electrons: the methods and resulting field theory are identical to those discussed in Ref. [28]. The renormalization group analysis shows that the $T=0$ transition can be either first or second order, depending upon the values of microscopic parameters. The gapped vison excitations in the $\mathrm{FL}^{*}$ state may be detected through the flux trapping experiments discussed in Ref. [29]. Furthermore, provided the transition is not too strongly first order, the presence of a critical charge $e$ bosonic mode implies that the su- 
perconducting state in the vicinity of this transition is a candidate for displaying stable $h c / e$ vortices [30,11].

Interesting physics obtains in the presence of an external uniform Zeeman magnetic field in the $\mathrm{FL}^{*}$ state. As the local moment and conduction electron systems are essentially decoupled in this phase, they both respond independently to the magnetic field. If the spinons are gapped in the fractionalized phase, then there would be a critical field $B_{c}$ associated with the onset of magnetization in the local moment system. Experimentally, this would be seen as a "metamagnetic" transition in the response of the system to an applied field. Interestingly, this onset transition could clearly be generically (i.e without any fine tuning) second order. Metamagnetic quantum criticality in strongly correlated systems has been the subject of some recent experimental 31] and theoretical studies [32], although accidental fine tuning has been invoked to obtain a second order transition.

This paper has established that metals with local moments in dimensions $d \geq 2$ can have non-magnetic ground states $\left(\mathrm{FL}^{*}\right)$ which are distinct from the familiar heavy Fermi liquid state (FL). The latter state has a Fermi surface enclosing a volume $\mathcal{V}_{F L}$ determined by the density, $\rho_{a}$, of both the conduction electrons and local moments; our topologically ordered $\mathrm{FL}^{*}$ state has sharp electronlike excitations on a Fermi surface enclosing a volume $\mathcal{V}_{F L^{*}}$ determined by $\left(\rho_{a}-1\right)$ (for $n_{\ell}=1$ this is the density of conduction electrons alone), along with additional 'fractionalized' excitations. In between these FL and $\mathrm{FL}^{*}$ states, a plethora of additional states associated with magnetic, superconducting, and charge order appear possible, along with non-trivial quantum critical points between them. We believe this rich phenomenology should find experimental realizations in the heavy fermion compounds.

We thank A. Chubukov, P. Coleman, M. P. A. Fisher, A. Georges, P. A. Lee, C. Pepin, and Q. Si for useful discussions. We were supported by the MRSEC program of the US NSF under grant number DMR-9808941 (T.S.), by US NSF Grant DMR 0098226 (S.S.), and by the DFG through SFB 484 (M.V.). T.S also acknowledges funding from the NEC Corporation and the hospitality of the Aspen Center for Physics.

[1] A. C. Hewson, The Kondo Problem to Heavy Fermions, (Cambridge University Press, Cambridge, 1993).

[2] P. Coleman, C. Pépin, Q. Si, and R. Ramazashvili, J. Phys: Condens. Matt. 13, 723 (2001).

[3] P. Coleman, cond-mat/0206003.
[4] H. Shiba and P. Fazekas, Prog. Theor. Phys. Supp. 101, 403 (1990); K. Ueda, T. Nishino, and H. Tsunetsugu, Phys. Rev. B 50, 612 (1994); S. Moukouri and L. G. Caron, Phys. Rev. B 54, 12212 (1996); P. Nozières, Eur. Phys. B 6, 447 (1998).

[5] M. Yamanaka, M. Oshikawa, and I. Affleck, Phys. Rev. Lett. 79, 1110 (1997).

[6] M. Oshikawa, Phys. Rev. Lett. 84, 3370 (2000).

[7] S. Burdin, A. Georges, and D. R. Grempel, Phys. Rev. Lett. 85, 1048 (2000).

[8] N. Andrei and P. Coleman, Phys. Rev. Lett. 62, 595 (1989).

[9] Yu. Kagan, K. A. Kikoin, and N. V. Prokof'ev, Physica B 182, 201-209 (1992).

[10] S. Burdin, D. R. Grempel, and A. Georges, Phys. Rev. B 66, 045111 (2002).

[11] T. Senthil and M. P. A. Fisher, Phys. Rev. B 62, 7850 (2000); Phys. Rev. B 63, 134521 (2001).

[12] N. Read and S. Sachdev, Phys. Rev. Lett. 66, 1773 (1991); X.-G. Wen, Phys. Rev. B 44, 2664 (1991).

[13] S. Kivelson, Phys. Rev. B 39, 259 (1989); N. Read and B. Chakraborty, Phys. Rev. B 40, 7133 (1989).

[14] N. E. Bonesteel, Phys. Rev. B 40, 8954 (1989).

[15] G. Misguich, C. Lhuillier, M. Mambrini, and P. Sindzingre, Eur. Phys. J. B 26, 167 (2002).

[16] For some sample geometries, it is necessary that the "adiabatic" flux insertion rate be faster than the energy splitting between the nearly degenerate ground states, which is exponentially small in system size.

[17] S. Doniach, Physica B 91, 231 (1977).

[18] P. W. Anderson, Science 235, 1196 (1987).

[19] N. Read and S. Sachdev, Phys. Rev. Lett. 62,1694 (1989).

[20] A fractionalized state is also possible for $n_{\ell}$ even, but in this case $\mathcal{V}_{F L^{*}}=\mathcal{V}_{F L}$, and so the Fermi volume no longer serves as a diagnostic for fractionalization.

[21] R. Moessner and S. L. Sondhi, Phys. Rev. Lett. 86, 1881 (2001).

[22] Fractionalized states in the model (11) were discussed recently by E. Demler, C. Nayak, H.-Y. Kee, Y.-B. Kim, and T. Senthil, Phys. Rev. B 65, 155103 (2002), but they did not notice that the conduction electrons will retain their bare quantum numbers at small $J_{K}$.

[23] This exotic superconductor is the $\mathrm{SC}^{*}$ state introduced in Ref. 11].

[24] E. Fradkin and S. H. Shenker, Phys. Rev. D 19, 3682 (1979).

[25] S. Sachdev and N. Read, Int. J. Mod. Phys. B 5, 219 (1991); M. Vojta, Y. Zhang, and S. Sachdev, Phys. Rev. B 62, 6721 (2000).

[26] S. Sachdev, Phys. Rev. B 45, 12377 (1992).

[27] S. Sachdev and J. Ye, Phys. Rev. Lett. 70, 3339 (1993).

[28] J. Ye and S. Sachdev, Phys. Rev. B 44, 10173 (1991).

[29] T. Senthil and M. P. A. Fisher, Phys. Rev. Lett. 86, 292 (2001).

[30] S. Sachdev, Phys. Rev. B 45, 389 (1992); N. Nagaosa and P. A. Lee, Phys. Rev. B 45, 966 (1992).

[31] S. A. Grigera et al., Science 294, 329 (2001).

[32] A. J. Millis et. al., Phys. Rev. Lett. 88, 217204 (2002). 